\title{
Resonant $x$-ray ptychographic nanotomography of kesterite solar cells
}

\author{
Giovanni Fevola $\odot,{ }^{1}$ Peter S. Jørgensen, ${ }^{1}$ Mariana Verezhak, ${ }^{2}$ Azat Slyamov $\odot,{ }^{1}$ Andrea Crovetto $\odot,{ }^{3,4}$ Zoltan I. Balogh ${ }^{5}$ \\ Christian Rein $\odot,{ }^{1}$ Stela Canulescu $\odot,{ }^{6}$ and Jens W. Andreasen $\odot^{1, *}$ \\ ${ }^{1}$ Department of Energy Conversion and Storage, Technical University of Denmark, DK-2800 Kgs. Lyngby, Denmark \\ ${ }^{2}$ Paul Scherrer Institute, CH-5232 Villigen PSI, Switzerland \\ ${ }^{3}$ Department of Physics, Technical University of Denmark, DK-2800 Kgs. Lyngby, Denmark \\ ${ }^{4}$ Helmholtz-Zentrum Berlin für Materialien und Energie, D-14109 Berlin, Germany \\ ${ }^{5}$ DTU Nanolab, Technical University of Denmark, DK-2800 Kgs. Lyngby, Denmark \\ ${ }^{6}$ Department of Photonics Engineering, Technical University of Denmark, DK-2800 Kgs. Lyngby, Denmark
}

(Received 31 December 2019; accepted 21 February 2020; published 30 March 2020)

\begin{abstract}
The $\mathrm{Cu}_{2} \mathrm{ZnSnS}_{4}$ kesterite is currently among the most promising inorganic, nontoxic, earth-abundant materials for a new generation of solar cells. Interfacial defects and secondary phases present in the kesterite active layer are, however, detrimental to the performance of the device. They are typically probed with techniques that are destructive or limited to the surface, and x-ray diffraction cannot reliably distinguish small amounts of zinc sulfide or copper tin sulfide from kesterite. Conversely, resonant ptychographic tomography, which relies on electron density contrast, overcomes these limitations. Here, we demonstrate how this technique can enable localization and quantification of secondary phases, along with measurements of adherence at the interfacial layers, on complete and functioning devices. In our experiment, we utilize an x-ray energy value far from absorption edges as well as three single energies corresponding to the absorption edges of $\mathrm{Cu}, \mathrm{Zn}$, and $\mathrm{Sn}$, to gain elemental sensitivity to these elements and enhance contrast between phases with similar electron density. As a result, we image and identify in the active layer grains of a secondary phase, namely, zinc sulfide, which is not easily discriminated by other standard characterization techniques. In addition, we are able to observe $\mathrm{Cu}$ diffused from the active layer into the $\mathrm{CdS}$ buffer layer as well as $\mathrm{Cu}$ in the form of copper sulfide at their interface. Other relevant morphological features are best resolved off-resonance at the optimal energy for the synchrotron beamline with $\sim 20 \mathrm{~nm}$ resolution.
\end{abstract}

DOI: 10.1103/PhysRevResearch.2.013378

\section{INTRODUCTION}

The $\mathrm{Cu}_{2} \mathrm{ZnSnS}_{4}$ (CZTS) kesterite is regarded among the most promising inorganic, earth-abundant, nontoxic materials to employ as a bulk active layer in a new generation of thin-film solar cells [1]. This quaternary compound is a direct semiconductor, with intrinsic $p$-type conductivity, and a suitable bandgap $(1.5 \mathrm{eV})$, which can also be tuned with Se alloying [2]. Besides these features, it shares with its successful parent chalcogenide technology of $\mathrm{Cu}(\mathrm{In}, \mathrm{Ga}) \mathrm{Se}_{2}$ (CIGS) a high theoretical efficiency (28\% for kesterite), but while efficiency above $20 \%$ has been demonstrated for CIGS, the current record for kesterite is around 12\% [3]. The lower performance of kesterite is largely attributed to the opencircuit voltage deficit, as outlined in previous studies [4]. The causes can be grouped into defects in the bulk kesterite [5], and defects at its interfaces with the buffer $\mathrm{CdS}$ layer [6] or with the $\mathrm{MoS}_{2}$ electrode [7]. In fact, the formation of pure kesterite is limited by a very unforgiving single-phase region

\footnotetext{
*jewa@dtu.dk

Published by the American Physical Society under the terms of the Creative Commons Attribution 4.0 International license. Further distribution of this work must maintain attribution to the author(s) and the published article's title, journal citation, and DOI.
}

and defect chemistry $[8,9]$ so that, in practice, secondary compounds (sulfides with different stoichiometry than CZTS) are likely to form together with the desired phase. It is well known that some secondary phases are more benign than others and that, for instance, it is preferable to grow kesterite in a Zn-rich environment, even though the effect on the nanoscale of these phases on a functioning device is not precisely known [2].

Some of the possible secondary phases are not easily detected. Cheng et al. were among the first to use SEM (scanning electron microscopy), Raman, and x-ray diffraction (XRD) to identify secondary phases in CZTS films [10]. They pointed out that although the main XRD peaks of CZTS, $\mathrm{ZnS}$, and $\mathrm{Cu}_{2} \mathrm{SnS}_{3}$ (CTS) overlap, one can identify three minor peaks that unambiguously characterize CZTS, but that does not rule out the potential presence of $\mathrm{ZnS}$ and CTS. The topic of detection and discrimination limits of secondary phases was investigated by Berg et al. [11], which highlighted that, besides complementing it with Raman, XRD can be further refined with Rietveld analysis. Even so, XRD can only discriminate these phases if they make up a large fraction of the overall film (at least $10 \% \mathrm{ZnS}$ and 50\% CTS). They also conclude that quantitative analysis is not possible for $\mathrm{XRD}$ and that $\mathrm{ZnS}$ is indiscernible for Raman with green light alone, even in ZnS-rich samples. In-depth Raman analysis [12] shows that with UV wavelengths $\mathrm{ZnS}$ is discerned due to the induced resonant vibrational modes. Likewise, Lafond et al. concluded that resonance of $\mathrm{Cu}$ and $\mathrm{Zn}$ is 
determining to enhance contrast for detection of the $\mathrm{Cu} / \mathrm{Zn}$ disorder with XRD [13]. The general conclusion by Kumar et al. is that "the defect concentration in CZTS is lower than the detectable limit for most techniques but sufficient enough for poor device performance" [9]. Hence, they emphasize the need in particular for novel characterization techniques and for a deeper understanding of defects and secondary phase formation.

Conventional techniques for secondary phase characterization are either destructive or are applied at an intermediate stage of the fabrication of the device. As the active layer is polycrystalline, with grains that can be larger than a micron, a full device should contain at least a few of these grains and be therefore at least a few microns in size. X-ray ptychographic tomography [14] makes it possible to quantitatively image a volume with a few tens of microns thickness and is thus uniquely suitable for a nanoscale investigation of a full device. The capabilities offered by the technique when applied to organic thin-film solar cells have been elucidated by previous experiments $[15,16]$. For the case of kesterite, however, despite the remarkable overall accuracy of the technique [17], secondary phases can lack sufficient contrast with respect to the main compound. In fact, $\mathrm{Cu}$ and $\mathrm{Zn}$ being neighboring elements in the periodic table, the relative difference in electron density can easily be below the limits of detection [17]. On the other hand, resonant x-ray ptychographic tomography (RXPT) [18] has been introduced to provide not only elemental sensitivity but also information on the chemical state. The method relies on the reduction in scattering power exhibited by a given element when the beam energy matches an absorption edge and, in its near-edge version, can be seen as the combination of x-ray absorption spectroscopy with ptychographic tomography. RXPT has already been applied to functional materials to detect a single element of interest $(\mathrm{Fe})$ [19] and its oxidation state [20]. More recently, RXPT was reported in the soft $\mathrm{x}$-ray regime, in the near-edge case (also referred to as spectroptychographic tomography) [21,22], and notably probing two different elemental edges $\left(\mathrm{Zn}-\mathrm{L}_{1}\right.$ and Al-K) [23]. Here we apply RXPT for the first time to a solar cell, where we illustrate that kesterite makes an interesting case, in which certain phases risk being overlooked by standard ptychographic tomography alone. In addition to the standard off-resonance energy, we probe three additional energies corresponding to characteristic X-ray absorption edges of $\mathrm{Cu}, \mathrm{Zn}$, and $\mathrm{Sn}$ in a wide energy range (4.5 to $9.7 \mathrm{keV}$ ), to gain elemental sensitivity to these elements and discriminate secondary phases. We demonstrate that alterations of electron density contrast are a means to highlight important features in solar devices.

\section{METHODS}

\section{A. Sample description and preparation}

The fabrication process of the investigated kesterite solar cells is described by Cazzaniga et al. [24]. The layer stack is a conventional architecture used in kesterite solar cells. Briefly, the precursors of CZTS are deposited on Mo-coated soda lime glass (SLG) substrates from ablation of a CZTS target in vacuum. The precursors grown by pulsed layer deposition (PLD) are annealed in a high-temperature sulfurized atmosphere, where the kesterite $(<450 \mathrm{~nm})$ and the $\mathrm{MoS}_{2}$ $(500 \mathrm{~nm})$ layers are formed. Subsequently, a CdS buffer layer $(60 \mathrm{~nm})$ is produced by chemical bath deposition (CBD) [25]; the intrinsic $\mathrm{ZnO}$ window layer $(50 \mathrm{~nm})$ and the Indium Tin oxide (ITO) contact layer $(200 \mathrm{~nm})$ are deposited by sputtering; an $\mathrm{MgF}_{2}$ anti-reflection coating $(100 \mathrm{~nm})$ is finally evaporated.

Two cylindrically shaped samples with a diameter of $5 \mu \mathrm{m}$ were prepared with the focused ion beam (FIB) lift-out technique [26] from the complete kesterite solar cells and mounted on OMNY pins [27]. Images from this process are available in the Supplemental Material [28], Fig. S1. The samples, hereafter referred to as E1 and E2, were milled out of two kesterite solar cells whose electrical parameters are given in Table S1. These two areas were intentionally selected from solar cells of significantly different efficiencies (1.6\% for E1, $0.8 \%$ for E2). The layer stack is a conventional geometry used in kesterite solar cells.

\section{B. Experimentals}

RXPT was carried out at the cSAXS beamline of the Swiss Light Source (SLS) at the Paul Scherrer Institute, Villigen, Switzerland, using the flOMNI setup described in detail elsewhere [29]. The energies for tomography were selected after an energy scan of ptychographic projections with $1 \mathrm{eV}$ resolution over a region of the kesterite active layer. The selected values are reported in Table I. For RXPT we used photon energies of $6.20 \mathrm{keV}$ (off-resonance), $8.99 \mathrm{keV}(\mathrm{Cu}$

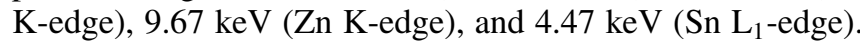
The sample was placed after the focal spot defined by a Fresnel Zone Plate [30] where the beam had a diameter of approximately $3 \mu \mathrm{m}$ for each energy. The ptychographic scans were performed with a $9 \times 3 \mu \mathrm{m}$ field of view $(\mathrm{H} \times \mathrm{V})$, following a Fermat spiral pattern [31] with a step size of $300 \mathrm{~nm}$. Exposure time was set to $0.1 \mathrm{~s}$ for all scans except for those at $4.47 \mathrm{keV}$, for which decreased flux was counteracted by doubling the exposure time. Scan duration was approximately 5 hours for each tomogram. Radiation damage was assessed by visual inspection of the three dimensional (3D) reconstructions and by cross-correlation alignment of the ptychographic projections versus measurement time. Despite the repeated measurement of each sample, there is no clear evidence of radiation damage on the scale of observation, although that cannot be ruled out on a finer scale (see note in Supplemental Material [28]).

The ptychographic projections were reconstructed using cSAXS Matlab code in an area of $800 \times 800$ pixels of the Eiger detector placed $5268 \mathrm{~mm}$ downstream from the sample, resulting in a pixel size and resolution stated in Table I using 300 iterations of the difference map algorithm [32] followed up by 500-900 iterations of a maximum likelihood refinement [33]. For tomography, 280 projections equally spaced in a 180-degree angular range were recorded. The phase of the reconstructed projections was used after post-processing alignment and removal of constant and linear phase components, and a modified filtered back projection algorithm was applied for the tomographic reconstruction [34,35]. The $3 \mathrm{D}$ resolution from Table I was determined by Fourier shell correlation (FSC) $[36,37]$ with the $1 / 2$-bit threshold criterion. 
TABLE I. Energies probed with RXPT and resolution assessment of the resulting tomograms.

\begin{tabular}{lcccc}
\hline \hline Edge & Off-resonance & Cu (K-edge) & Zn (K-edge) & Sn (L1-edge) \\
\hline Symbol & $\lambda_{\text {off }}$ & $\lambda_{\mathrm{Cu}}$ & $\lambda_{\mathrm{Zn}}$ & $\lambda_{\mathrm{Sn}}$ \\
Energy [keV] & 6.201 & 8.986 & 9.666 & 4.468 \\
Electron Binding Energy in Natural Form [keV] & - & 8.979 & 9.659 & 18.77 \\
Voxel size [nm] & 17.55 & 25.24 & 18.77 & 24.36 \\
3D resolution from FSC [nm] & 17.55 & 25.98 & 37 & 27.54 \\
2D resolution based on line profiling [nm] & 26 & 31 & 37 \\
\hline \hline
\end{tabular}

The FSC method over an interior region of the whole stack results in values close to single pixel resolution due to very high contrast features with very sharp interfaces (e.g., $\mathrm{MgF}_{2}$ ITO or Mo- $\mathrm{MoS}_{2}$ interface). However, based on averaged line profiles over 3 cuts across the Mo-MoS 2 interface (also see Fig. S2), we obtain slightly worse estimates of resolution reported in Table I.

\section{Theoretical basis}

The theory behind resonant ptychographic tomography is described in its first experimental demonstration [18] and can be summed up as follows. Tomography measures the sample as a volume of complex refractive indices $n=1-$ $\delta+j \beta$, where $\beta$ relates to the absorption and $\delta$ to the phase shift of the single voxel. The dependence on the wavelength of refractive indices can be traced back to the atomic scattering factor $f_{0}=f_{1}+j f_{2}$, and they are related as follows:

$$
\begin{aligned}
n(\lambda) & =1-\delta(\lambda)-j \beta(\lambda) \\
& =1-\frac{r_{0}}{2 \pi} \lambda^{2} \sum_{k} n_{\mathrm{at}}^{k}\left[f_{1}^{k}(\lambda)+j f_{2}^{k}(\lambda)\right],
\end{aligned}
$$

where $k$ refers to the $k$ th atomic species, $n_{\text {at }}$ is the atomic density of such species, $r_{0}$ is the classical electron radius, and $j$ is the imaginary unit. $f_{1}$ and $f_{2}$ are the dispersion corrections to the atomic scattering factor that modifies both phase and absorption contrast of a voxel. RXPT relies on the fact that $f_{1}$ exhibits a dip in the proximity of resonant edges, whereas it equals the atomic number of the element otherwise. If the $k$ th element is the only one close to a resonant edge, its atomic density can be obtained by subtracting tomograms measured on-resonance and off-resonance:

$$
n_{\mathrm{at}}^{k}=\frac{1}{\Delta f_{1}^{k}} \frac{2 \pi}{r_{0}}\left(\frac{\delta_{\mathrm{off}}}{\lambda_{\text {off }}^{2}}-\frac{\delta_{\text {on }}}{\lambda_{\text {on }}^{2}}\right),
$$

where $\Delta f_{1}^{k}=f_{1}^{k}\left(\lambda_{\text {off }}\right)-f_{1}^{k}\left(\lambda_{\text {on }}\right)$. Although the measurements taken are in principle sufficient to determine the atomic density of elements in kesterite (including S), in our case the precise determination of the atomic density is not the optimal strategy to distinguish between phases, as the factor $\Delta f_{1}^{k}$ can be strongly affected by element speciation. Besides, even assuming pure kesterite, the $\mathrm{Cu}$ and $\mathrm{Zn}$ edges are not distant enough to assume that $\mathrm{Cu}$ 's contribution cancels at $\mathrm{Zn}$ edge and vice versa. In symbols, with respect to $\delta$, Eq. (1) in our case becomes

$$
\begin{aligned}
& \delta_{\text {off }}=\frac{r_{0}}{2 \pi} \lambda^{2}\left[n_{\mathrm{at}}^{\mathrm{Cu}} f_{1}^{\mathrm{Cu}}\left(\lambda_{\text {off }}\right)+n_{\mathrm{at}}^{\mathrm{Zn}} f_{1}^{\mathrm{Zn}}\left(\lambda_{\text {off }}\right)+n_{\mathrm{at}}^{\mathrm{Sn}} f_{1}^{\mathrm{Sn}}\left(\lambda_{\text {off }}\right)+n_{\mathrm{at}}^{\mathrm{S}} f_{1}^{\mathrm{S}}\left(\lambda_{\text {off }}\right)\right], \\
& \delta_{\mathrm{Cu}}=\frac{r_{0}}{2 \pi} \lambda^{2}\left[n_{\mathrm{at}}^{\mathrm{Cu}} f_{1}^{\mathrm{Cu}}\left(\lambda_{\mathrm{Cu}}\right)+n_{\mathrm{at}}^{\mathrm{Zn}} f_{1}^{\mathrm{Zn}}\left(\lambda_{\mathrm{Cu}}\right)+n_{\mathrm{at}}^{\mathrm{Sn}} f_{1}^{\mathrm{Sn}}\left(\lambda_{\mathrm{Cu}}\right)+n_{\mathrm{at}}^{\mathrm{S}} f_{1}^{\mathrm{S}}\left(\lambda_{\mathrm{Cu}}\right)\right],
\end{aligned}
$$

where $\delta_{\mathrm{Cu}}$ is the on-resonance measurement at the $\mathrm{Cu}$ edge. When subtracting the two, the term $n_{\mathrm{at}}^{\mathrm{Zn}}\left(f_{1}^{\mathrm{Zn}}\left(\lambda_{\text {off }}\right)-f_{1}^{\mathrm{Zn}}\left(\lambda_{\mathrm{Cu}}\right)\right)$ can hardly be neglected, and if included adds its own uncertainty to the sum.

Therefore, in our analysis, we refer to the quantity

$$
n_{\mathrm{e}}(\lambda)=\frac{2 \pi \delta(\lambda)}{\lambda^{2} r_{0}},
$$

to examine contrast variations at the different energies. $n_{\mathrm{e}}$ provides an indirect, optical measurement of the electron density of the material. Such measurement closely matches the actual electron density far from absorption edges, whereas it gives a lower, apparent electron density in proximity of the edges. Differently from $\delta, n_{\mathrm{e}}$ accounts for the strong $\sim \lambda^{2}$ dependence and can therefore be compared for the four energies. An apparent electron density lower than the expected one signals the presence of a resonating element.

\section{Data post-processing}

The expected values of $\delta$ or $n_{\mathrm{e}}$ can be accurately computed with Eq. (1) using an experimental scattering factor database [38] when resonance does not occur. These predictions give an excellent match with measured values (see section Results and Discussion). For instance, $\mathrm{ZnS}$ and CZTS values, which are the only easily noticeable peaks in the bulk layer histogram differ by less than $2 \%$. It is a more complicated matter to predict the expected $\delta$ /apparent density of secondary phases if they contain elements for which the absorption edge is shifted due to possibly different oxidation state and specific near edge fine structure. Therefore, the following procedure is conceived to scan tomograms for secondary phases and identify them.

First, we consider the list of secondary phases that are most likely to be found in kesterite. We predict the phase and absorption contrast using Eq. (1), as shown in the Supplemental Material [28]. 

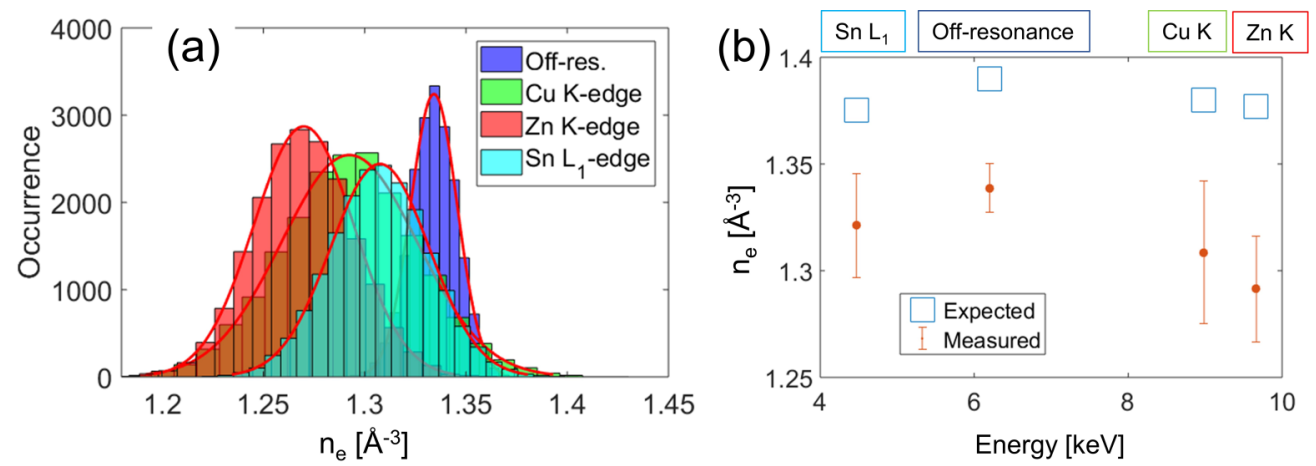

FIG. 1. Histograms of electron density value for voxels in a $\mathrm{MoS}_{2}$ slice of E1 (a) and comparison with calculated $\delta$ of $M o S_{2}$ from tabulated values (b). The measured values are about $95 \%$ of the expected ones.

Then, for any given secondary phase:

(1) The expected value of electron density is computed for all tomograms which are off-resonance for that phase. For example, for $\mathrm{Cu}_{3} \mathrm{SnS}_{4}$ we predict the expected $n_{\mathrm{e}}\left(\lambda_{\text {off }}\right)$ and $n_{\mathrm{e}}\left(\lambda_{\mathrm{Zn}}\right)$.

(2) Voxels with those expected values are segmented out by thresholding. The dispersions computed from the $\mathrm{MoS}_{2}$ analysis (see below) are used to define the upper and lower threshold, with a coverage factor of 3. For example, for $\mathrm{Cu}_{3} \mathrm{SnS}_{4}$ we consider the voxels that fit the range of the predicted values in the tomograms measured at $\lambda_{\text {off }}$ and $\lambda_{\mathrm{Zn}}$. Because of similar electron density, other phases (CZTS) mainly, might also be included in this range.

(3) Multivariate histograms of the segmented voxels are computed to highlight different peaks and associations. For example, for $\mathrm{Cu}_{3} \mathrm{SnS}_{4}$, histograms of $n_{\mathrm{e}}\left(\lambda_{\mathrm{Cu}}\right)$ and $n_{\mathrm{e}}\left(\lambda_{\mathrm{Sn}}\right)$ are computed for the voxels previously segmented. $\mathrm{Cu}_{3} \mathrm{SnS}_{4}$ can be distinguished from CZTS at this point because of its higher $\mathrm{Cu}$ atomic density and therefore lower $n_{\mathrm{e}}\left(\lambda_{\mathrm{Cu}}\right)$.

The tomograms of E1 and E2 were interpolated to the largest voxel size of the available tomograms at different energies. That corresponds to $25 \mathrm{~nm}$ of the $\mathrm{Cu}$ tomogram for E1 and of the Sn tomogram for E2. The interpolation is made with an FFT-based function and the subsequent alignment with a subpixel registration procedure [39].

\section{E. Uncertainty}

The outstanding quantitativeness of ptychographic nanotomography has been discussed by Diaz et al. [17]. Because of upgrades to the beamline and because the resonant scans are taken in different conditions, we need to reevaluate uncertainty. We have used the same approach as Diaz et al. to assess quantitativeness of our measurements. Absolute values for dispersion of measurements can be assumed to be independent of the average measured values. Therefore, such deviation can be measured in any uniform volume, including air, and then used as tolerance for thresholding around an expected value.

The $\mathrm{MoS}_{2}$ layer is used as a reference to estimate the uncertainty of each tomogram. The hypothesis, in this case, is that it is a relatively uniform layer and shows little or no subvoxel porosity. The distribution of measured electron density is displayed in Fig. 1. The measurements are numerous enough to fit well a Gaussian. Means and standard deviation are summed up in Table II. The illustrated statistics refer to sample E1, but the same values are also found in E2, confirming reproducibility of the measurements. The values computed according to Eq. (1) fit the trend of the slight dependence with energy. The measured values suggest an average of $5 \pm 1 \%$ subvoxel porosity of the layer. It is worth pointing out in this instance the error bias of reporting the electron density of a set of voxels on histograms, as voxels can be partially filled by material whereas they cannot be over-filled. Therefore histograms are more likely to be overpopulated on the low end than the high, leading to underestimation of the average value.

\section{RESULTS AND DISCUSSION}

Two solar cells (herein referred to as E1 and E2) were inspected and prepared with focused ion beam (FIB) as $5 \mu \mathrm{m}$ in diameter and $2 \mu \mathrm{m}$ minimal height cylindrical samples for tomography. They were imaged with a 3D resolution between 18 and $37 \mathrm{~nm}$ at four $\mathrm{x}$-ray energies. The slices and volume rendering we report here display the indirect optical measurement $n_{\mathrm{e}}$ of the electron density of materials, which is in general what ptychography provides in off-resonance conditions. However, elemental resonance induces a reduction in the scattering power of given elements, which results in the measurement of a lower apparent electron density. For further details about samples and the theoretical basis for our experiment refer to Sec. II. The results are organized in three subsections presenting, respectively: morphological features observed in off-resonance tomograms; chemical features highlighted by the combined analysis with resonant phase tomograms; a comparison between phase and absorption-based tomograms. The other two subsections include a discussion of the obtained results as well as a brief outlook on future experiments.

\section{A. Morphological features}

As expected from previous studies, the whole layered structure is accurately resolved by ptychographic nanotomography. As illustrated by Fig. 2(a), there is sufficient contrast between all adjacent layers. The kesterite layer has a nominal electron density close to that of $\mathrm{CdS}$ and $\mathrm{MoS}_{2}$ (see Table S2), but even a rough threshold-based segmentation 
TABLE II. Average measured electron density in $\mathrm{MoS}_{2}$ slice comprising ca. 20000 voxels. Off-resonance, the dispersion of values is less than $1 \%$ of the measured value and is below $3 \%$ in all cases. The small mean values measured in the air surrounding the sample are subtracted from the whole tomogram to correct for nonzero-mean noise.

\begin{tabular}{|c|c|c|c|c|c|}
\hline & Mean & Standard deviation & Percent deviation & Air & Air-adjusted \\
\hline & {$\left[\AA^{-3}\right]$} & {$\left[\AA^{-3}\right]$} & {$[\%]$} & {$\left[\AA^{-3}\right]$} & {$\left[\AA^{-3}\right]$} \\
\hline Off-resonance & 1.33 & 0.012 & 0.86 & -0.005 & 1.34 \\
\hline Cu K-edge & 1.29 & 0.034 & 2.60 & -0.017 & 1.31 \\
\hline Zn K-edge & 1.27 & 0.025 & 1.94 & -0.023 & 1.29 \\
\hline Sn $\mathrm{L}_{1}$-edge & 1.31 & 0.024 & 1.86 & -0.014 & 1.32 \\
\hline
\end{tabular}

can distinguish the three layers. The only exception is the couple of layers between the active kesterite layer and the ITO electrode layer. Even in the higher resolution tomograms, the $\mathrm{CdS} n$-type layer and the electron transport $\mathrm{ZnO}$ layer, which

\section{(a)}
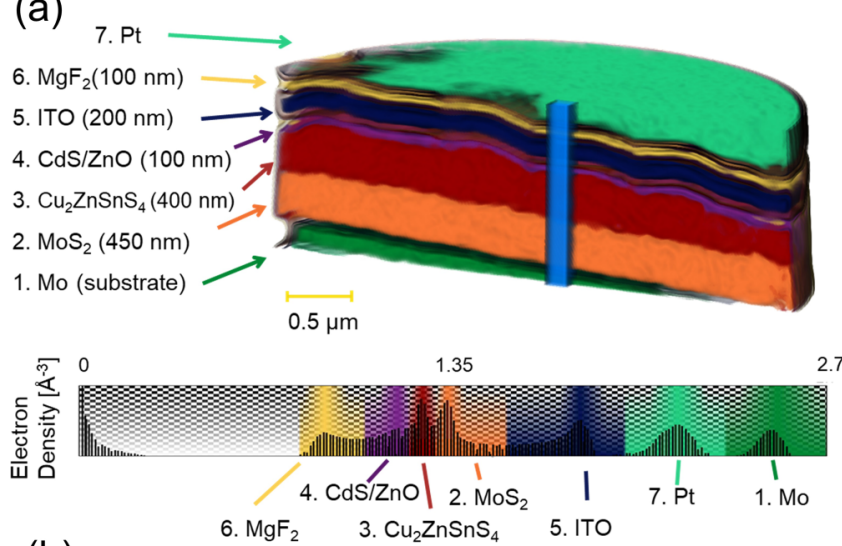

(b)

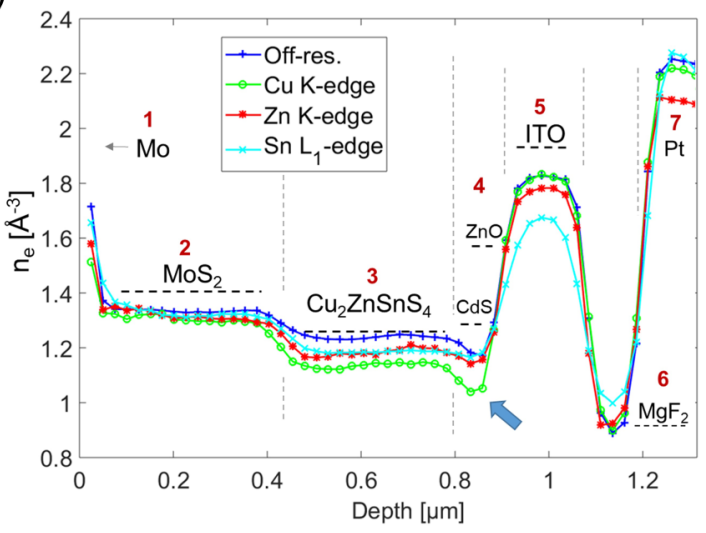

FIG. 2. (a) Volume rendering of the layer stack from the resulting tomographic reconstruction of sample E1. Layers are enumerated in ascending order from bottom to top and their expected size are reported in parenthesis. False colors are assigned as in the colorbar to the main peaks of the histogram of electron densities to distinguish between the layers. (b) Apparent electron density profile of E1 sample at the different energies. The profile is evaluated across the blue slab in the center of (a), averaging on an area of $8 \times 8$ pixels $\left(200 \times 200 \mathrm{~nm}^{2}\right)$. Profile measurements can be compared to the actual electron densities of the compounds (dashed black lines), also reported in Table $\mathrm{S} 2$. The area is chosen within a regular grain, with flat surface and no additional features. The dip of electron density in proximity of the $\mathrm{CdS}$ layer measured at $\mathrm{Cu}$ edge is interpreted as evidence of $\mathrm{Cu}$ migration into the layer. are both around $50 \mathrm{~nm}$ in thickness and have been observed with SEM [24], cannot be clearly distinguished at any energy, but they are instead somehow mixed in a single layer of 4-5 voxels. Most importantly, although the electron density of $\mathrm{CdS}$ and particularly of $\mathrm{ZnO}$ is not lower than that of kesterite, a 4-5 voxels thin double layer appears with a lower electron density compared to kesterite. This is shown on the crosssectional profile plot in Fig. 2(b), where a distinguishable dip in electron density between the kesterite and the ITO layers can be observed. Based on observations at other energies, we later elaborate on the analysis of this layer.

The profile plot also illustrates a relatively good match between measured and expected electron density of the layers (Table S2). The slightly lower values for the $\mathrm{MoS}_{2}$ and the ITO layers can be attributed to variation in the stoichiometry and/or to subvoxel porosity. For ITO in particular, the apparent electron density of this layer measured at the Sn edge is lower because of In, which presents an appreciable decrease of scattering power at that energy. For the off-resonance tomogram, the distribution of electron densities as measured on each voxel follows that of the phase $\delta$, which is reported later when it is compared to that of the absorption $\beta$.

Figure 3 shows morphological features in the obtained tomograms. Our analysis focuses on the active layer (kesterite) and its interfaces with $\mathrm{CdS}$ and $\mathrm{MoS}_{2}$. The interface between kesterite and $\mathrm{MoS}_{2}$ (the latter being formed as well during the sulfurization process) appears to be very smooth and there is no particular detail to highlight aside from a couple of pinholes [d1 in Fig. 3(a)]. Other features visible in our tomograms include a discontinuity in the ITO layer (d2), thinning of the CZTS layer (d3), and some larger CdS precipitates in the buffer layer (d4) due to the CBD process. These features can be observed in the movie $\mathrm{S} 1$ provided in Supplemental Material [28].

Figure 3(b) depicts the morphology of the grains in the upper part of the kesterite layer. With a layer thickness of ca. $400 \mathrm{~nm}$ and a voxel size of $25 \mathrm{~nm}$, the layer is imaged in 16 slices. From the 4 top slices shown in Fig. 3(b), it can be noticed that the grain contours appear better defined in the higher part of the layer, where grain separation increases. Grain contours can be observed at all energies, although they are evidently best resolved at the off-resonance energy because of the better resolution, as shown in Fig. 3(c). Individual grains can be distinguished due to the presence of wider grain boundaries, i.e., voids considerably wider than the lattice parameter. In fact, although we refer to them as grains, they are likely to contain subgrains with different lattice orientations but without any gap. For the latter there will be no 
(a)

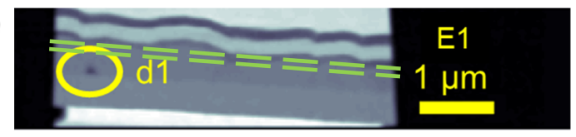
E1 d2 $\approx$

(b)
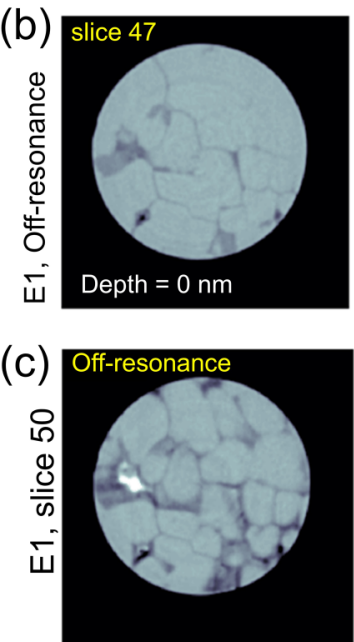

Depth $=25 \mathrm{~nm}$
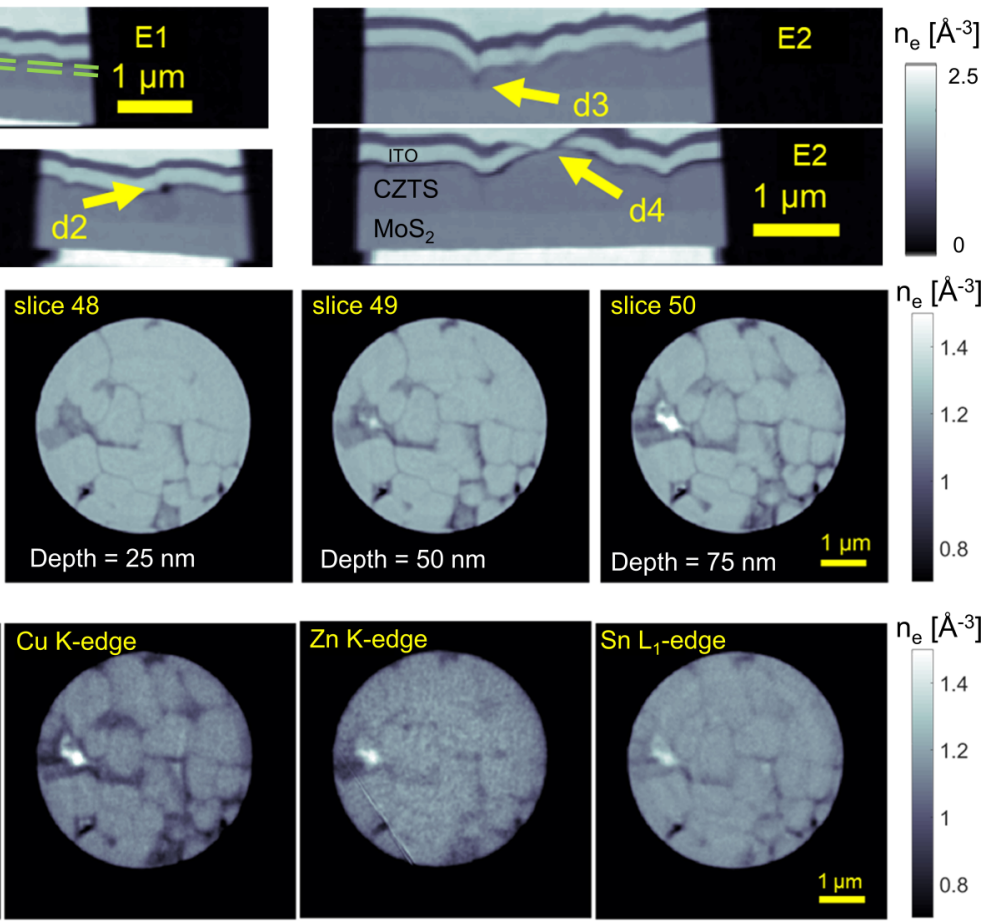

FIG. 3. Morphological features from phase tomograms of the CZTS layer. (a) Sagittal slices of E1 and E2 highlighting different types of defects: pinholes (d1), CdS precipitates (d2), thinning of CZTS layer (d3), discontinuity of ITO (d4). The dashed green lines in E1 indicate the range of depth of axial slices illustrated next. (b) Grain morphology of the active layer illustrated by four consecutive axial slices of the active layer, from bottom (slice 47) to top (slice 50). Distance between each slice equals the voxel size (25 $\mathrm{nm}$ ). Grain size is around $1 \mu \mathrm{m}$ and the grain boundaries are clearly resolvable. (c) A comparison of the same slice at different edge energies showing that morphological features are best highlighted at the off-resonance energy. The colorbar is truncated in (b) and (c) to enhance gray level contrast.

appreciable change in electron density on the nanometer scale (ca. $20 \mathrm{~nm}$ ) and the structure would appear homogeneous at the experimental resolution. Aside from sporadic voids, the lower part of the kesterite layer appears compact up to the voxel level, and the well-defined grain contours fade although a grain separation still exists. As previously observed with SEM, average grain lateral size is around $1 \mu \mathrm{m}$ [24]. For a $3 \mathrm{D}$ view of the grain morphology in E1 refer to movie S2 of Supplemental Material [28].

\section{B. Chemical features}

Differences between aligned tomograms recorded at different energies reveal information about secondary phases. The CZTS layer correctly displays a decreased electron density compared to the off-resonance case, confirming that the scan energy at every resonant edge has been correctly selected. The measured electron density off-resonance is $1.237 \AA^{-3}$, in excellent agreement with the $1.246 \AA^{-3}$ calculated with Eq. (1) (see Sec. II) and with the $1.26 \AA^{-3}$ deduced from material properties (Table $\mathrm{S} 2$ ). This value is shifted down to $1.117,1.142,1.167 \AA^{-3}$ when measured at $\mathrm{Cu}, \mathrm{Zn}$, and $\mathrm{Sn}$ edge-energies, respectively, which in all cases are $10 \%$ below the original value. The shift is clearest at the $\mathrm{Cu}$ edge because $\mathrm{Cu}$ is the most abundant element in kesterite.

Following previous characterization results [24], it is reasonable to assume that most of the active layer's composition is pure CZTS, and if secondary phases are present they are likely to be a minority given our measurements of the device performance. The most legitimate objection to this argument is that most of the bulk layer (\#3 in Fig. 2) might be CTS, which still can perform as a decent absorber material and has virtually the same density as kesterite. However, relying on our data, that is ruled out by the fact that most of the bulk layer exhibits a decrease in apparent electron density when scanned at the Zn edge, whereas CTS would not, being $\mathrm{Zn}$-free. Some chemical features are depicted in Fig. 4. The most evident difference between the energy tomograms are some grains of a few hundred $\mathrm{nm}$ in E2 and three larger ones in E1. They extend roughly from bottom to top of the active layer in height, and they have a size of up to $500 \mathrm{~nm}$. A 3D view of these grains and their localization in the active layer of E1 is available in movie S2. As shown in Fig. S3 and Table S3, these grains are identifiable as $\mathrm{ZnS}$. The difference between the on- and off-resonance measurements increases at $\mathrm{Zn}$ edge and the electron density measured off-resonance is compatible with the expected values of this phase. $\mathrm{SnS}_{2}$ phase would also be possible but is excluded in this case as no inversion of contrast with kesterite at $\mathrm{Cu}$ edge is observed (see Fig. S3). Instead, contrast with the main phase is decreased at $\mathrm{Cu}$ - and $\mathrm{Sn}$ - edges, which is as expected for $\mathrm{ZnS}$.

The bivariate histograms of Fig. 5 illustrate the case of $\mathrm{ZnS}$. These histograms are computed including voxels with electron density within the predicted range of $\mathrm{ZnS}$, at all energies except for the Zn-edge energy. Porous voxels of other material at interfaces or at grain boundaries also fall within this range. Therefore, when the correlation between apparent (on-resonance) and actual (off-resonance) electron densities 


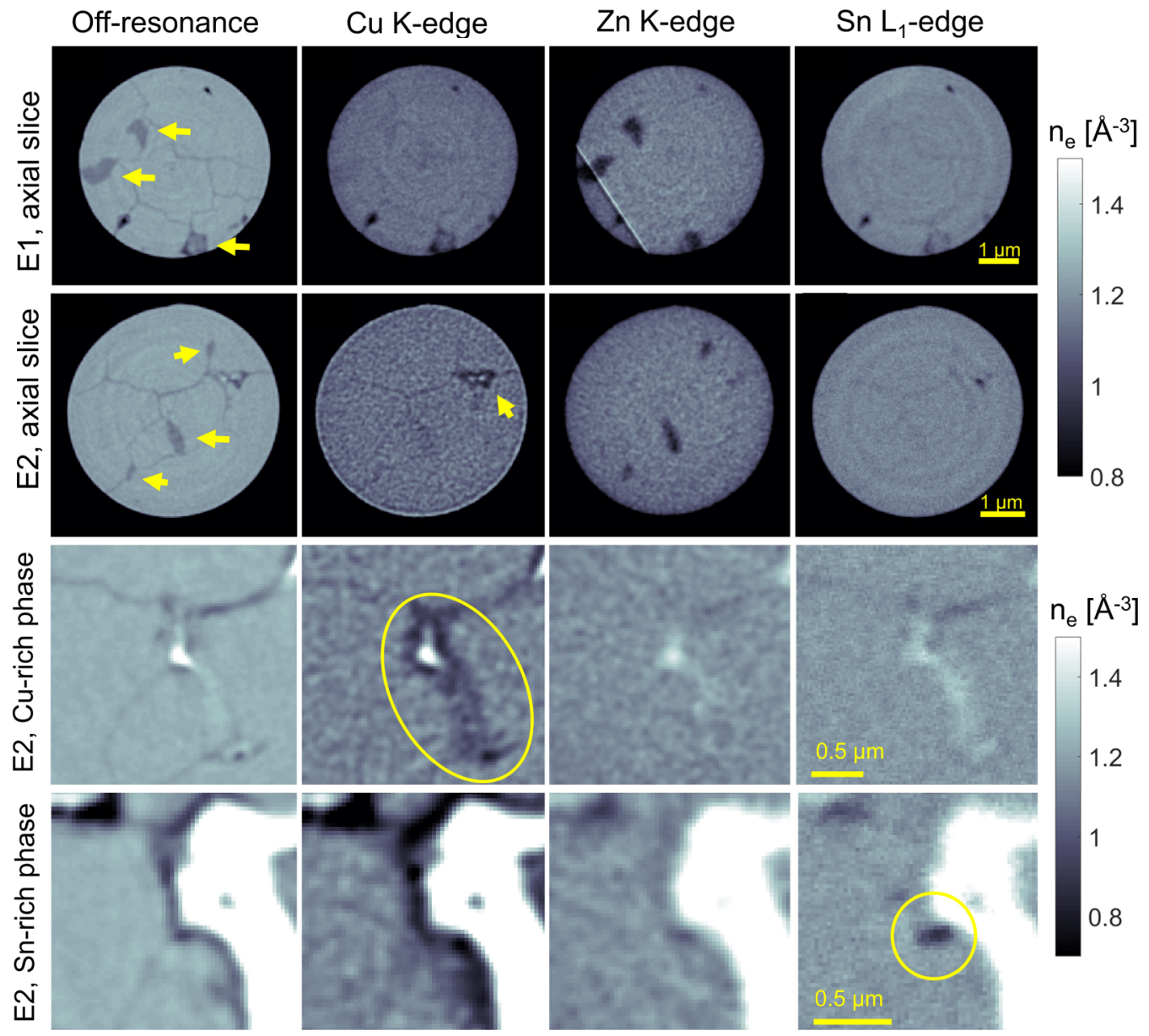

FIG. 4. Chemical features revealed by contrast variation at different energies. Each row reports the same slice or area at the four different energies (columns). ZnS grains (yellow arrows) are found in E1 and E2 (row 1 and 2). Cu- and Sn-rich clusters (circled in yellow) from E2 are highlighted in row 3 and 4, respectively. Colorbars are truncated to enhance gray level contrast.

is evaluated two clusters appear. The larger one relates to CZTS, whereas the smaller one relates to $\mathrm{ZnS}$. If supported (a)

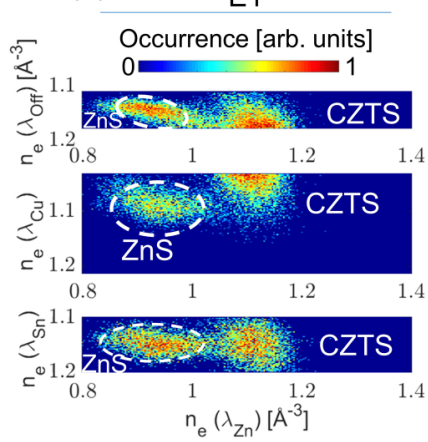

(b)

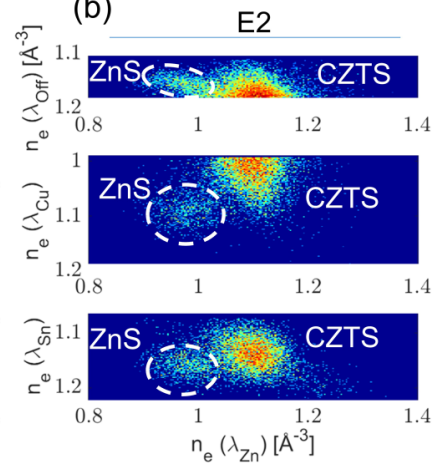

FIG. 5. Bivariate histograms of $\mathrm{ZnS}$ in $\mathrm{E} 1$ (a) and E2 (b). The apparent electron density at the Zn edge is reported on the $x$ axis vs. the other energies. The active layer of E1 contains significantly larger amounts of $\mathrm{ZnS}$. Clusters in the top row plots are asymmetrical because of the lower dispersion off-resonance. The apparent electron density of $\mathrm{ZnS}$ is shifted down to ca. $0.95 \AA^{-3}$ at the $\mathrm{Zn}$-edge. Expected shift values are reported in Table S3 of Supplemental Material [28]. by enough statistics so that they can be clearly identified, the centroids of the $\mathrm{ZnS}$ clusters could provide also the information of zero atomic density of $\mathrm{Cu}$ and $\mathrm{Sn}$, which can in principle support a more detailed analysis ${ }^{1}$ (see subsection Theoretical Basis in METHODS). The continuum of points connecting the ZnS small cluster to the main cluster of CZTS relates to voxels at the grain boundaries, which are better resolved at the off-resonance energy. In this case, the higher detail mixes CZTS with ZnS points and gives the impression that the cluster are better separated with the on-resonance tomograms in spite of their lower quality. Moreover, as $\mathrm{Zn}$ is more present in ZnS than in CZTS, the distance between the two clusters' centroids is in fact increased, which corresponds to the desired effect of contrast enhancement. We note that even in the case of $\mathrm{E} 1$, where entire grains of $\mathrm{ZnS}$ are present, the total volume in the two samples is $<2.5 \%$ of total CZTS volume $\left(0.13 \mu \mathrm{m}^{3} \mathrm{ZnS}\right.$ versus $5.13 \mu \mathrm{m}^{3}$ CZTS in E1). These levels are below the detection limit for XRD [11].

${ }^{1}$ If $\mathrm{ZnS}$ is assumed, then $n_{\mathrm{at}}^{\mathrm{Cu}}=n_{\mathrm{at}}^{\mathrm{Sn}}=0$ can be set in the system of Eqs. (3), which becomes in this case overdetermined, having four equations and only two unknowns, allowing for an experimental indirect measurement of the scattering factor corrections. 

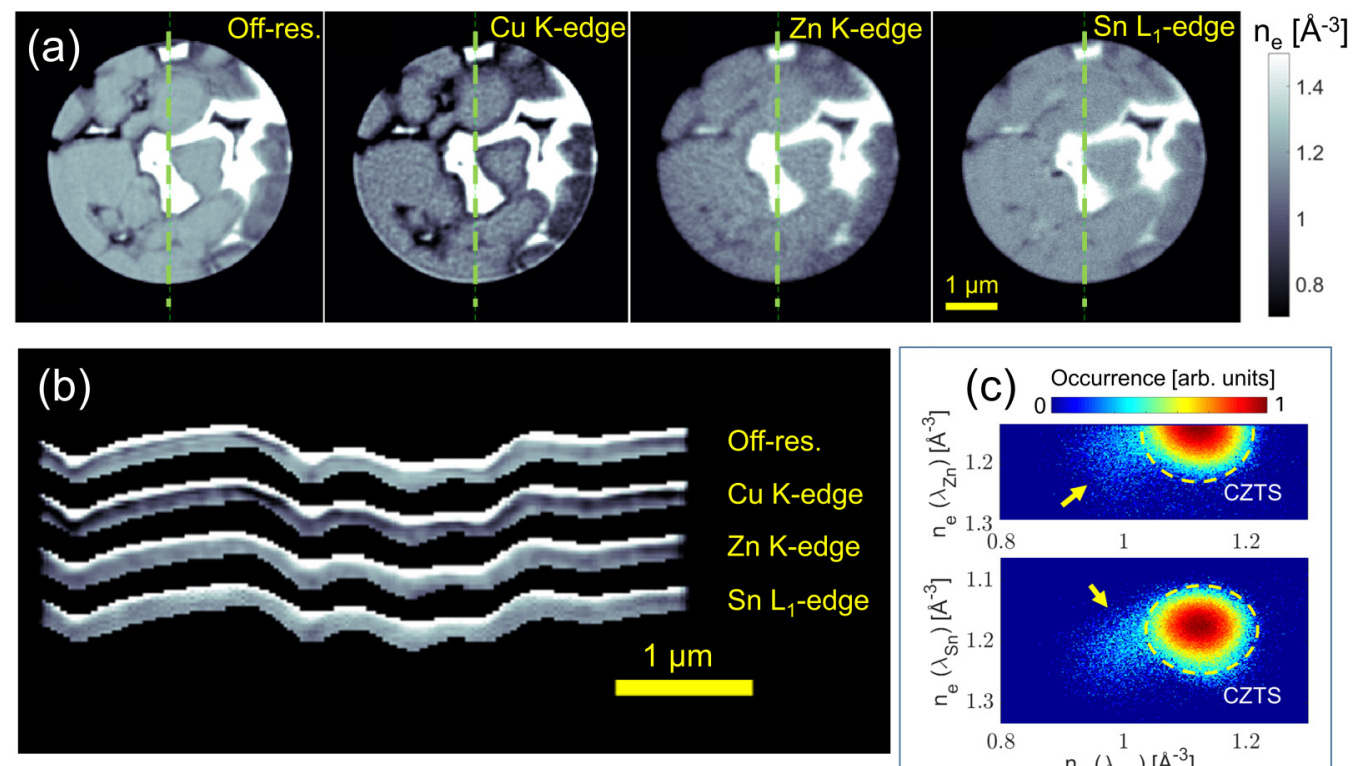

(C) Occurrence [arb. units]

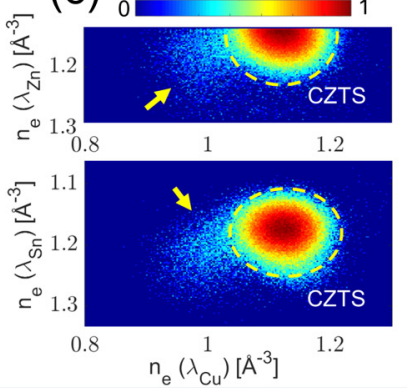

FIG. 6. Images of the CdS/CZTS interface. (a) Axial slice of E2 at the four different energies. The dashed green line refers to the cross section of the segmented interface in (b). The image taken at the $\mathrm{Cu}$ K-edge appears darker than the others highlighting Cu migration into the CdS layer. That is illustrated by the trail of points (yellow arrows) around the CZTS cluster in the bivariate histograms of (c). These points show a lower electron density at the Cu edge and similar or higher density at $\mathrm{Zn}$ or Sn edge (nominal electron density of CdS is $1.28 \AA^{-3}$ ).

Figure 6 shows the contrast enhancement of the interfacial $\mathrm{CdS}$ layer at the $\mathrm{Cu} \mathrm{K}$-edge. All the grain boundaries appear thicker from all axial views of previous figures. When segmented, the CdS layer shows lower apparent electron density at the $\mathrm{Cu} \mathrm{K}$-edge, highlighting the presence of $\mathrm{Cu}$. The resonant effect of $\mathrm{ZnO}$ within this layer is hardly noticeable. From the profile plot of Fig. 3(b), after the kesterite layer, the profile lines would rejoin the off-resonance line if there were no resonant elements, but instead the dip continues for the profile recorded at the $\mathrm{Cu}$ edge. Further evidence of the same phenomenon is shown in Fig. S4 of Supplemental Material on a wider stretch of the CdS layer.

Other evident differences are observed at the other resonant energies (Fig. 4). Closer to the interface, some spots are identified where electron density is slightly higher than kesterite off-resonance, while it is decreased at the $\mathrm{Cu}$ edge and in the off-resonance absorption-based tomogram. This behavior suggests the presence of $\mathrm{CuS}$ or $\mathrm{Cu}_{3} \mathrm{SnS}_{4}$ phases. The lack of these features in other tomograms is consistent with the depicted contrast scenario (cf. Figs. S3 and S5).

\section{Absorption versus phase-based tomograms}

Absorption $(\beta)$-based tomograms provide valuable redundancy or additional information to the observations from the phase $(\delta)$ tomograms, that allows discrimination between different phases of similar electron density. While the phase contrast exhibits a dip around the resonant edge, absorption is increased, implying a substantially different contrast scenario (see Fig. S5 in Supplemental Material [28]). As the quality of absorption-based tomograms is significantly inferior to that of phase tomograms at these energies, both with respect to resolution and to quantitativeness, their information alone is insufficient to reliably discriminate small aggregates of secondary phases and would yield numerous false positives. Nevertheless, in Fig. 7 the absorption-based tomogram clarifies the picture of the $\mathrm{Cu}$-rich aggregate described in Fig. 4. Since it has lower $\beta$ values than kesterite, the $\mathrm{Cu}$-rich aggregate cannot be $\mathrm{Cu}_{3} \mathrm{SnS}_{4}$ (cf. Fig. S5) nor $\mathrm{CdS}$ with diffused $\mathrm{Cu}(\mathrm{CdS}$ has approximately double the absorption coefficient of kesterite). The $\mathrm{ZnS}$ grains in the two samples can also be identified in the bivariate histograms of absorption versus phase (Fig. S6).

\section{Discussion}

Given the important, still unclear role of grain boundaries in kesterite solar cells, the ability to resolve them is crucial. It has been reported that they help charge transport of electrons to the electrode, and secondary phases, not all detrimental, can be found in their proximity [9]. However, Ito's review [2] concludes that results from theoretical [40] and experimental [41] investigations on the role of grain boundaries in kesterites are not fully consistent, but lean toward its benign nature as in CIGS or CdTe devices, rather than a detrimental one like in Si devices. Therefore, we believe the ability to clearly resolve them off-resonance with tomographic methods could have significant impact. It is also shown, however, that grain boundaries still remain a subvoxel feature. Although it would be of interest to compare grain boundaries at different energies, it would be inconclusive on the scale of the achieved experimental resolution.

The single-voxel uncertainty estimated empirically is significantly below the expected 5\% uncertainty reference [17] and makes it possible to distinguish most secondary phases only based on a prediction of electron density. Even with a coverage factor of 2 (confidence interval of 95\%), the uncertainty is below $\pm 2 \%$ (for details see Sec. II). Based on 

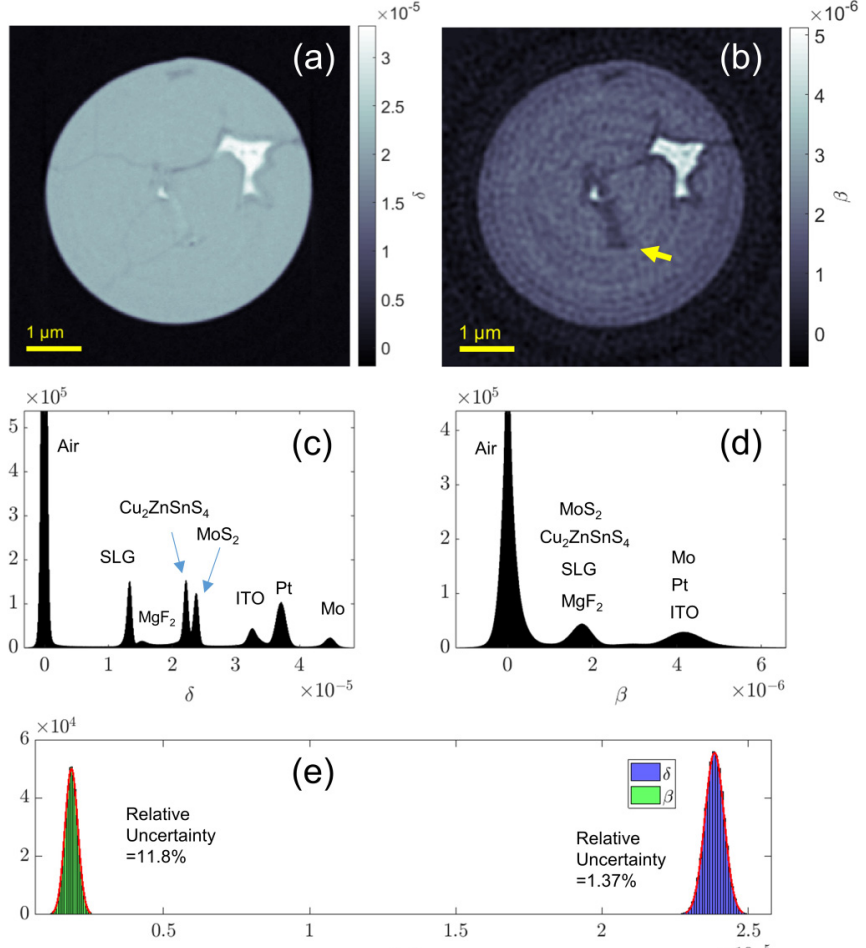

(e)

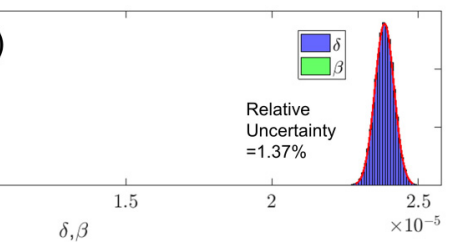

FIG. 7. Top row: comparison of an axial slice of the active layer of E2, phase $\delta$ (a) vs. absorption $\beta$ (b) off-resonance tomograms. The slice contains a feature (yellow arrow) illustrated in Fig. 4. In this case the dark feature in the absorption-based tomogram rules out $\mathrm{Cu}_{3} \mathrm{SnS}_{4}$ in favor of $\mathrm{CuS}$. The higher contrast of phase tomograms is illustrated by the histogram of occurrences of $\delta$ (c) and $\beta$ (d) in the total volume. The dispersion of measured values is small enough for the $\delta$ to allow high contrast between layers (c), whereas $\beta$ values are roughly centered around two values only (d). In (e) the dispersion of measured values is quantified on a slice of $\mathrm{MoS}_{2}$. The dispersion of measurement is only slightly higher for $\delta$ values, which are an order of magnitude higher than $\beta$, providing superior relative accuracy.

that, $\mathrm{Cu}_{3} \mathrm{SnS}_{4}, \mathrm{Sn}_{2} \mathrm{~S}_{3}$, and $\mathrm{SnS}_{2}$ are the only phases that do not show sufficient contrast with respect to pure kesterite. However, without elemental sensitivity it is not possible to distinguish a subvoxel porosity (voids, gaps between grains) from lower density inherent to the secondary phases. Unlike the large grains found in $\mathrm{E} 1$, a few $\mathrm{ZnS}$ particles found in E2 are sufficiently small to be mistaken for porous kesterite or voids. In that case, the resonant tomogram at $\mathrm{Zn}$ edge provides definitive evidence about their nature. It also occurs that a kesterite grain can fit well within the $\mathrm{Sn}_{2} \mathrm{~S}_{3}$ range, and in that case the resonant tomogram at the $\mathrm{Cu}$-edge excludes it.

The biggest defects in the interfacial layer (bubbles with diameter up to ca. $200 \mathrm{~nm}$ ) are due to the CdS deposition step. These are CdS precipitates arising from the CBD process. Besides, although only a few, some grains are spaced enough from each other to allow CdS to fill this gap (see also Fig. S4). Although this can in principle short-circuit the $\mathrm{CdS}$ and $\mathrm{MoS}_{2}$ layers, there is no electrical contact between the layers, as an open circuit voltage different than zero has been measured in both cells.
$\mathrm{Cu}$ migration into the $\mathrm{CdS}$ layer has been reported in literature $[42,43]$, and it has also been argued that $\mathrm{Cu}$ has a crucial role in determining the performance of the CdS/kesterite interface [44]. Therefore, it is of interest to quantify our observations with respect to the interface. The lower electron density of the CdS layer can be attributed to subvoxel porosity or in principle to $\mathrm{Cu}$ substitution, as $\mathrm{Cu}$ is significantly lighter than $\mathrm{Cd}$. The weight of each factor is not easy to estimate, considering that thickness of this layer is barely above the resolution. Porosity alone would imply a value of $8 \%$ in E1 and $11 \%$ in $\mathrm{E} 2$. The amount of $\mathrm{Cu}$ could in principle be estimated by Eq. (2) (see Methods), if the scattering factor of $\mathrm{Cu}$ were available. The role of $\mathrm{Cu}$ in decreasing the electron density is supported by the fact that a lower value is observed concurrently with a higher $\mathrm{Cu}$ presence, which is what the comparison between E1 and E2 suggests. As no post-annealing was performed on this cell, $\mathrm{Cu}$ migration must have occurred in the CBD step. This same observation is reported by Kim et al. [44], who noted a lower performance in a cell with intermixing of interfacial layers, while the reasons for that were unclear.

$\mathrm{ZnS}$ presence appeared less sporadic than expected. A grain of similar size as those in E1 $(500 \mathrm{~nm})$ was observed in a previous investigation of ours in a sample prepared from the same device. Thus, these $\mathrm{ZnS}$ grains, rather than an exception, here appear as the rule. As they can escape quantification and localization with XRD [11], they were not detected in the previous characterization. Moreover, they can qualify as a further responsible for the performance gap previously outlined [24]. The performance gap was attributed to the nonuniformity of the ITO layer, which has also been observed here, but only in one instance. As kesterite is grown in a Zn-rich environment, this finding is not unexpected.

On the contrary, the observation of a CuS aggregate was not expected. $\mathrm{CuS}$ droplets were observed before as a byproduct of the PLD step [24]. These droplets could be removed by KCN-etching, but that was judged as unnecessary, as they seemed to disappear after annealing. It was also previously shown that the appearance of the film changes radically after annealing, and it is assumed that all the excess $\mathrm{Cu}$ is absorbed into the kesterite layer. Our findings show that part of it could still form $\mathrm{Cu}$-rich phases other than kesterite. A portion of the aggregate is also compatible with $\mathrm{Cu}_{3} \mathrm{SnS}_{4}$ values, but this option seems to be ruled out by the absorption-based tomograms.

Only two of the Sn-rich phases shown in Fig. 4 have been found in the two samples. They are about $100 \mathrm{~nm}$ in size, therefore larger than the assessed resolution, and they are arguably not artifacts as they are systematically obtained in different reconstructions. However, they do not match any of the most likely Sn-rich phases summarized in Table S3. To be identified as $\mathrm{SnS}_{2}$ or $\mathrm{Sn}_{2} \mathrm{~S}_{3}$, they would have to show significantly enhanced contrast at $\mathrm{Cu}$ energy, which does not appear to be the case. The absorption-based tomograms do not help in this respect because of the small size of the feature. As Sardashti et al. have observed $\mathrm{SnO}_{2}$ after air annealing [42], this hypothesis has to be considered, but the electron density of $\mathrm{SnO}_{2}$, over $1.8 \AA^{-3}$, is significantly higher than that of kesterite and would be evident at off-resonance energies. Although very unlikely to form [45], we have to consider 
$\mathrm{Cu}_{2} \mathrm{ZnSn}_{3} \mathrm{~S}_{8}$ as a possibility as it should have similar resonant behavior as kesterite at all energies except than at the Sn edge. However, the easier interpretation is that of a subvoxel mix of particles rich of $\mathrm{Sn}_{x} \mathrm{~S}$ with $1<x<2$, which would explain why nothing is visible off-resonance.

It is unclear whether we can relate our findings on a $5 \mu \mathrm{m}$-diameter cylinder to efficiency measurement of the whole tested area. Nonetheless, we note that E1 had higher efficiency than E2 (1.6\% versus $0.8 \%)$. The overall comparison shows that $\mathrm{E} 1$ contains significantly more $\mathrm{ZnS}$, but no $\mathrm{Cu}$ - or Sn-rich secondary phases. Besides, it shows less $\mathrm{Cu}$ at the interface than E2, a more uniform thickness of the active layer (although smaller grains), and no discontinuity in the $\mathrm{CdS} / \mathrm{ZnO}$ nor in the ITO layer.

\section{E. Outlook}

The only element of kesterite whose resonant edge has not been probed is S. Sulfur K-edge is around $2.5 \mathrm{keV}$ and is outside of the capabilities of the beamline. However, with our experimental design, it would not provide much new information as all secondary phases are sulphides and the $S$ density contrast is supposedly not higher than the offresonance electron density contrast. Beamlines that can probe such low energy are typically devoted to soft x-ray techniques, and therefore are unable to probe the energies of $\mathrm{Cu}$ and $\mathrm{Zn}$ or samples of this size. However, kesterite systems have extensively been studied around the S K-edge with near edge spectroscopy $[46,47]$. An imaging experiment at the S K-edge could exploit the near edge fine structure provided by different oxidation states of the elements within different phases rather than by atomic densities.

To draw conclusions on the smallest features, one should ideally have the same resolution and accuracy at all energies. Doubling the exposure time has led in the case of the Sn edge to an improvement of quantitativeness and resolution. As kesterite is known for its stability and our samples tolerated a dose of four tomograms, it can be beneficial to increase the exposure time also in the other cases to reduce the dispersion of measurements and allow detection of clusters in bivariate histograms that are even closer than the illustrated cases. It is also useful to point out that there exists a trade-off between resolution and quantitativeness. Defects of bigger size and lower contrast could be highlighted by binning/interpolation. Further increase of quantitativeness is particularly desirable for automatic analysis of data, as it would determine better success rate for segmentation.

Arguably, more interesting observations can be made at interfaces on a scale finer than tens of nanometers, which could be observed with electron microscopy methods. However, these methods limit sample size well below the typical grain size in kesterite. Compared to other synchrotron techniques, RXPT probes a larger scale than XRD but provides real space information, and has superior resolution and accuracy for quantification than 3D fluorescence even in its most advanced correlative version [48]. Elemental sensitivity, which is the main advantage of fluorescence, is obtained in this case for the elements of interest at the cost of a change of energy and additional scans. Although 3D information can in principle be obtained with fluorescence, tomographic reconstruction might pose major challenges due to self absorption [49] and would not achieve a resolution comparable to RXPT. The most important feature of this technique is the possibility for in situ or operando studies. The impact of these defects and features can be quantified with measurements of x-ray beam induced current or voltage (XBIC/XBIV [50-52]), which is the natural extension of this study. The missing requirements for such studies are the ability to electrically contact the prepared samples and a different scheme for the ptychographic scan. The first requirement is easily overcome by a laminographic stage $[53,54]$, suitable for planar devices and which does not require milling to micron size. The second requirement is simply met by placing the sample closer to the focus point, so that the measured XBIC/XBIV map can have a resolution closer to the focus size. We foresee this approach to have a massive impact for the characterization of photovoltaic materials, especially with the introduction of fourth-generation synchrotrons.

\section{CONCLUSIONS}

We have used RXPT to image in 3D a full kesterite solar cell on the nanoscale. We demonstrated the capability of the technique to detect not only morphological features, such as grain boundaries, pinholes, thinning of layers, but also chemical features such as migration of $\mathrm{Cu}$ into the $\mathrm{CdS}$ layer and presence of secondary phases that are not easily detected with conventional techniques. Secondary phases are mapped here thanks to a different contrast mechanism, based on electron density, and to alterations of contrast induced by elemental resonance. Importantly, the ability to discriminate phases is only limited by spatial resolution, i.e., as long as a $\mathrm{ZnS}$ particle is larger than $40 \mathrm{~nm}$ it can be detected independently of the percentage of the total volume, enabling examination of state-of-the-art rather than defective devices. As the technique enables imaging of morphological and chemical features relevant for performance of solar devices in a nondestructive way, it potentially allows for in situ or operando studies. In combination with XBIC measurement and a laminographic stage, it can be used to determine the impact of nanoscale defects on the overall performance of solar devices.

\section{ACKNOWLEDGMENTS}

We thank the staff of the Swiss Light Source (SLS) at the Paul Scherrer Institute (PSI) for the support with RXPT experiments. X-ray nanotomography experiments were carried out at the cSAXS beamline, Paul Scherrer Institute, Switzerland. This study was supported by the European Research Council (ERC) under the European Union's Horizon 2020 research and innovation programme (SEEWHI Consolidator Grant No. ERC-2015-CoG-681881) and by the Ministry of Higher Education and Science (DANSCATT Grant No. 705500007B). M.V. acknowledges funding from SNSF Grant No. 200021L_169753. M.V. and A.C. acknowledge financial support from the European Union's Horizon 2020 research and innovation program under the Marie Skłodowska-Curie Grant Agreements No. 701647 (M.V.) and No. 840751 (A.C.). 
[1] S. Giraldo, Z. Jehl, M. Placidi, V. Izquierdo-Roca, A. PérezRodríguez, and E. Saucedo, Adv. Mater. 31, 1806692 (2019).

[2] K. Ito, Copper Zinc Tin Sulfide-Based Thin-Film Solar Cells (John Wiley \& Sons Ltd, Chichester, UK, 2014).

[3] S. K. Wallace, D. B. Mitzi, and A. Walsh, ACS Energy Lett. 2, 776 (2017).

[4] S. Siebentritt, Thin Solid Films 535, 1 (2013).

[5] S. Levcenko, J. Just, A. Redinger, G. Larramona, S. Bourdais, G. Dennler, A. Jacob, and T. Unold, Phys. Rev. Appl. 5, 024004 (2016).

[6] A. Crovetto, A. Cazzaniga, R. B. Ettlinger, J. Schou, and O. Hansen, Sol. Energy Mater. Sol. Cells 187, 233 (2018).

[7] P. D. Antunez, S. Li, D. M. Bishop, D. B. Farmer, T. S. Gershon, J. B. Baxter, and R. Haight, Appl. Phys. Lett. 113, 033903 (2018).

[8] I. D. Olekseyuk, I. V. Dudchak, and L. V. Piskach, J. Alloys Compd. 368, 135 (2004).

[9] M. Kumar, A. Dubey, N. Adhikari, S. Venkatesan, and Q. Qiao, Energy Environ. Sci. 8, 3134 (2015).

[10] A.-J. Cheng, M. Manno, A. Khare, C. Leighton, S. A. Campbell, and E. S. Aydil, J. Vac. Sci. Technol. A Vacuum, Surfaces, Film. 29, 051203 (2011).

[11] D. M. Berg, M. Arasimowicz, R. Djemour, L. Gütay, S. Siebentritt, S. Schorr, X. Fontané, V. Izquierdo-Roca, A. Pérez-Rodriguez, and P. J. Dale, Thin Solid Films 569, 113 (2014).

[12] X. Fontané, L. Calvo-Barrio, V. Izquierdo-Roca, E. Saucedo, A. Pérez-Rodriguez, J. R. Morante, D. M. Berg, P. J. Dale, and S. Siebentritt, Appl. Phys. Lett. 98, 181905 (2011).

[13] A. Lafond, L. Choubrac, C. Guillot-Deudon, P. Fertey, M. Evain, and S. Jobic, Acta Crystallogr. Sect. B Struct. Sci. Cryst. Eng. Mater. 70, 390 (2014).

[14] M. Dierolf, A. Menzel, P. Thibault, P. Schneider, C. M. Kewish, R. Wepf, O. Bunk, and F. Pfeiffer, Nature 467, 436 (2010).

[15] E. B. L. Pedersen, D. Angmo, H. F. Dam, K. T. S. Thydén, T. R. Andersen, E. T. B. Skjønsfjell, F. C. Krebs, M. Holler, A. Diaz, M. Guizar-Sicairos, D. W. Breiby, and J. W. Andreasen, Nanoscale 7, 13765 (2015).

[16] H. F. Dam, T. R. Andersen, E. B. L. Pedersen, K. T. S. Thydén, M. Helgesen, J. E. Carlé, P. S. Jørgensen, J. Reinhardt, R. R. Søndergaard, M. Jørgensen, E. Bundgaard, F. C. Krebs, and J. W. Andreasen, Adv. Energy Mater. 5, 1400736 (2015).

[17] A. Diaz, P. Trtik, M. Guizar-Sicairos, A. Menzel, P. Thibault, and O. Bunk, Phys. Rev. B 85, 020104(R) (2012).

[18] C. Donnelly, M. Guizar-Sicairos, V. Scagnoli, M. Holler, T. Huthwelker, A. Menzel, I. Vartiainen, E. Müller, E. Kirk, S. Gliga, J. Raabe, and L. J. Heyderman, Phys. Rev. Lett. 114, 115501 (2015).

[19] J. Ihli, A. Diaz, Y. Shu, M. Guizar-Sicairos, M. Holler, K. Wakonig, M. Odstrcil, T. Li, F. Krumeich, E. Müller, W.-C. Cheng, J. Anton van Bokhoven, and A. Menzel, J. Phys. Chem. C 122, 22920 (2018).

[20] Y.-S. Yu, M. Farmand, C. Kim, Y. Liu, C. P. Grey, F. C. Strobridge, T. Tyliszczak, R. Celestre, P. Denes, J. Joseph, H. Krishnan, F. R. N. C. N. C. Maia, A. L. D. D. Kilcoyne, S. Marchesini, T. P. C. Leite, T. Warwick, H. Padmore, J. Cabana, and D. A. Shapiro, Nat. Commun. 9, 921 (2018).

[21] M. Hirose, N. Ishiguro, K. Shimomura, N. Burdet, H. Matsui, M. Tada, and Y. Takahashi, Angew. Chemie 130, 1490 (2018).
[22] J. Wu, X. Zhu, M. M. West, T. Tyliszczak, H.-W. Shiu, D. Shapiro, V. Berejnov, D. Susac, J. Stumper, and A. P. Hitchcock, J. Phys. Chem. C 122, 11709 (2018).

[23] J. Wu, X. Zhu, D. A. Shapiro, J. R. I. Lee, T. Van Buuren, M. M. Biener, S. A. Gammon, T. T. Li, T. F. Baumann, and A. P. Hitchcock, J. Phys. Chem. C 122, 25374 (2018).

[24] A. Cazzaniga, A. Crovetto, C. Yan, K. Sun, X. Hao, J. Ramis Estelrich, S. Canulescu, E. Stamate, N. Pryds, O. Hansen, and J. Schou, Sol. Energy Mater. Sol. Cells 166, 91 (2017).

[25] G. Hodes, Chemical Solution Deposition of Semiconductor Films (CRC Press, Boca Raton, FL, 2002).

[26] R. M. Langford and C. Clinton, Micron 35, 607 (2004).

[27] M. Holler, J. Raabe, R. Wepf, S. H. Shahmoradian, A. Diaz, B. Sarafimov, T. Lachat, H. Walther, and M. Vitins, Rev. Sci. Instrum. 88, 113701 (2017).

[28] See Supplemental Material at http://link.aps.org/supplemental/ 10.1103/PhysRevResearch.2.013378 for: SEM images from sample preparation; electrical characterization of devices; resolution assessment through line profiling; electron density of compounds; expected contrast simulations; sagittal chemical view of CdS buffer layer filling; bivariate histogram of absorption-based tomograms; note on radiation damage; movie $\mathrm{S} 1$, sagittal slice view of sample E1 (MPG); movie S2, volume rendering of sample E1 (MPG).

[29] M. Holler, J. Raabe, A. Diaz, M. Guizar-Sicairos, C. Quitmann, A. Menzel, and O. Bunk, Rev. Sci. Instrum. 83, 073703 (2012).

[30] M. Odstrčil, M. Lebugle, M. Guizar-Sicairos, C. David, and M. Holler, Opt. Express 27, 14981 (2019).

[31] X. Huang, H. Yan, R. Harder, Y. Hwu, I. K. Robinson, and Y. S. Chu, Opt. Express 22, 12634 (2014).

[32] P. Thibault, M. Dierolf, A. Menzel, O. Bunk, C. David, and F. Pfeiffer, Science 321, 379 (2008).

[33] P. Thibault and M. Guizar-Sicairos, New J. Phys. 14, 063004 (2012).

[34] M. Guizar-Sicairos, A. Diaz, M. Holler, M. S. Lucas, A. Menzel, R. A. Wepf, and O. Bunk, Opt. Express 19, 21345 (2011).

[35] M. Guizar-Sicairos, J. J. Boon, K. Mader, A. Diaz, A. Menzel, and O. Bunk, Optica 2, 259 (2015).

[36] M. Van Heel and M. Schatz, J. Struct. Biol. 151, 250 (2005).

[37] R. P. J. Nieuwenhuizen, K. A. Lidke, M. Bates, D. L. Puig, D. Grünwald, S. Stallinga, and B. Rieger, Nat. Methods 10, 557 (2013).

[38] S. Sasaki, Numerical Tables of Anomalous Scattering Factors Calculated by the Cromer and Liberman's Method (Tsukuba, Japan, 1989).

[39] M. Guizar-Sicairos, S. T. Thurman, and J. R. Fienup, Opt. Lett. 33, 156 (2008).

[40] J. Li, D. B. Mitzi, and V. B. Shenoy, ACS Nano 5, 8613 (2011).

[41] J. B. Li, V. Chawla, and B. M. Clemens, Adv. Mater. 24, 720 (2012).

[42] K. Sardashti, R. Haight, T. Gokmen, W. Wang, L.-Y. Chang, D. B. Mitzi, and A. C. Kummel, Adv. Energy Mater. 5, 1402180 (2015).

[43] F. Liu, C. Yan, J. Huang, K. Sun, F. Zhou, J. A. Stride, M. A. Green, and X. Hao, Adv. Energy Mater. 6, 1600706 (2016).

[44] J. Kim, S. Park, S. Ryu, J. Oh, and B. Shin, Prog. Photovoltaics Res. Appl. 25, 308 (2017).

[45] E. A. Pogue and A. A. Rockett, in Conf. Rec. IEEE Photovolt. Spec. Conf. (IEEE, Piscataway, NJ, 2016), pp. 471-475. 
[46] J. Just, C. M. Sutter-Fella, D. Lützenkirchen-Hecht, R. Frahm, S. Schorr, and T. Unold, Phys. Chem. Chem. Phys. 18, 15988 (2016).

[47] J. Just, D. Lützenkirchen-Hecht, O. Müller, R. Frahm, and T. Unold, APL Mater. 5, 126106 (2017).

[48] J. Deng, Y. H. Lo, M. Gallagher-Jones, S. Chen, A. Pryor, Q. Jin, Y. P. Hong, Y. S. G. Nashed, S. Vogt, J. Miao, and C. Jacobsen, Sci. Adv. 4, eaau4548 (2018).

[49] M. Stuckelberger, B. West, T. Nietzold, B. Lai, J. M. Maser, V. Rose, and M. I. Bertoni, J. Mater. Res. 32, 1825 (2017).

[50] C. Ossig, T. Nietzold, B. West, M. Bertoni, G. Falkenberg, C. G. Schroer, and M. E. Stuckelberger, J. Vis. Exp. 150, e60001 (2019).
[51] B. M. West, M. Stuckelberger, H. Guthrey, L. Chen, B. Lai, J. Maser, V. Rose, W. Shafarman, M. Al-Jassim, and M. I. Bertoni, Nano Energy 32, 488 (2017).

[52] M. E. Stuckelberger, T. Nietzold, B. M. West, B. Lai, J. M. Maser, V. Rose, and M. I. Bertoni, in 2017 IEEE 44th Photovolt. Spec. Conf. (IEEE, Piscataway, NJ, 2017), pp. 2179-2184.

[53] M. Holler, M. Odstrcil, M. Guizar-Sicairos, M. Lebugle, E. Müller, S. Finizio, G. Tinti, C. David, J. Zusman, W. Unglaub, O. Bunk, J. Raabe, A. F. J. Levi, and G. Aeppli, Nat. Electron. 2, 464 (2019)

[54] M. Odstrcil, M. Holler, J. Raabe, and M. Guizar-Sicairos, in Image Sens. Technol. Mater. Devices, Syst. Appl. V, edited by N. K. Dhar and A. K. Dutta (SPIE, Bellingham, WA, 2018), p. 28. 